\title{
Zuber's Coronavirus Disease (COVID- 19) Standards for Hospitals
}

\author{
Zuber Mujeeb Shaikh \\ Director, Corporate Quality Improvement, Patient Safety and Risk Management \\ Dr. Sulaiman Al-Habib Medical Group, \\ Riyadh-11643, Kingdom of Saudi Arabia \\ ORCID-ID- https://orcid.org/0000-0002-1491-8379 \\ E-mail: drzuber5@yahoo.co.in
}

Area/Section: Health Sciences.

Type of the Paper: Conceptual Research.

Type of Review: Peer Reviewed as per $|\mathrm{C}| \mathrm{O}|\mathrm{P}| \mathrm{E} \mid$ guidance.

Indexed in: OpenAIRE.

DOI: http://doi.org/10.5281/zenodo.4757071

Google Scholar Citation: IJHSP

\section{How to Cite this Paper:}

Shaikh, Zuber Mujeeb, (2021). Zuber's Coronavirus Disease (COVID- 19) Standards for Hospitals. International Journal of Health Sciences and Pharmacy (IJHSP), 5(1), 103-116. DOI: http://doi.org/10.5281/zenodo.4757071.

International Journal of Health Sciences and Pharmacy (IJHSP)

A Refereed International Journal of Srinivas University, India.

Crossref DOI : https://doi.org/10.47992/IJHSP.2581.6411.0064

(C) With Author.

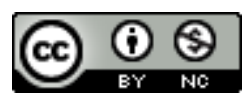

This work is licensed under a Creative Commons Attribution-Non-Commercial 4.0 International License subject to proper citation to the publication source of the work.

Disclaimer: The scholarly papers as reviewed and published by the Srinivas Publications (S.P.), India are the views and opinions of their respective authors and are not the views or opinions of the SP. The SP disclaims of any harm or loss caused due to the published content to any party. 


\title{
Zuber's Coronavirus Disease (COVID- 19) Standards for Hospitals
}

\author{
Zuber Mujeeb Shaikh \\ Director, Corporate Quality Improvement, Patient Safety and Risk Management \\ Dr. Sulaiman Al-Habib Medical Group, \\ Riyadh-11643, Kingdom of Saudi Arabia \\ ORCID-ID- https://orcid.org/0000-0002-1491-8379 \\ E-mail: drzuber5@yahoo.co.in
}

\begin{abstract}
Purpose: The Coronavirus Disease (COVID-19) is affecting 220 nations and territories in the world. As of May 11th, 2021, there were 158,651,638 reported and confirmed cases of COVID-19 to WHO, out of which 3,299,764 were reported death to WHO. However, there are no defined, structured and concise Coronavirus Disease (COVID- 19) Standards for Hospitals in order to manage such cases in the hospitals by maintain quality, patient safety, risk management and patient experience.

Objective: To develop Coronavirus Disease (COVID- 19) Standards for Hospitals.

Method: This is a review of literature study and the data were collected with comprehensive searches in the online databases of goggle scholars and research gate.

Conclusions: The study concluded with "Zuber's Coronavirus Disease (COVID- 19) Standards for Hospitals."
\end{abstract}

Keywords: Coronavirus Disuse, COVID-19, Hospital, Standards

\section{INTRODUCTION :}

Coronavirus Disease 2019 (COVID-19), also known as the coronavirus, or COVID, is a transmissible illness caused by Severe Acute Respiratory Syndrome Coronavirus 2 (SARS-CoV-2). The first known case was identified in Wuhan, China, in December 2019 [1]. The disease has since spread worldwide, leading to an ongoing pandemic [2]. The control of coronavirus disease (COVID-19) outburst is an international concern.

The Coronavirus Disease (COVID-19) is affecting 220 nations and territories in the world. As of May 11th, 2021, there were 158,651,638 reported and confirmed cases of COVID-19 to WHO, out of which $3,299,764$ were reported death to WHO. However, there are no defined, structured and concise Coronavirus Disease (COVID- 19) Standards for Hospitals in order to manage such cases in the hospitals by maintain quality, patient safety, risk management and patient experience.

\section{REVIEW OF LITERATURE :}

There is global shortage of resources to fight the deadly contagious disease COVID-19 mostly in the lowincome countries [3]. The world's wealthiest countries have been gripped by resource shortages, including shortages of personal protective equipment (PPE) and ventilators, during the coronavirus disease 2019 (COVID-19) pandemic [4,5]. Leaders of the healthcare organization should implement the measures to create positive attitude of protection and care towards their employees in order to cope up with the new work schedules, and to build effective team relationships despite of several challenges. Simultaneously, leaders should focus of the goals and objectives of the organization and ensure that quality improvement, patient safety, risk management and patient experience are pivotal in their all services [6]. Infection control and prevention problems are the most commonly found deficiencies in nursing homes, even in the highest CMS rated nursing homes [7]. Multiple vulnerabilities in nursing homes have been reported widely during the COVID-19 pandemic including: [8, 9, 10, 11, 12, 13, 14, 15].

a) Insufficient staffing

b) Lack of acceptable personal protective equipment (PPE) and compliance to the use of PPE's

c) Unreliable hand hygiene

d) Inappropriate isolation techniques 
e) Unreliable sanitization of equipment

f) Poor tracking of infections

g) High staff turnover and Staff working while ill

In particular, inadequate staffing and lack of a stable workforce has been identified as a weak point in nursing homes [16].

The researchers have revealed that the societies should use the advantage of Information and Technology to sustenance in an emergency management [17]. Current challenges include low sensitivity for test results as well as limited staff and resources [18]. The researchers have revealed that the import and local manufacture of medical oxygen gas to ensure its uninterrupted supply to designated COVID-hospitals is important and should be identified by the local administration [19].

Researchers had revealed that accreditation showed positive impact on hospital emergency transport services (Shaikh, 2016) [20], Department Services (Shaikh, 2017) [21], Dietary Services (Shaikh, 2017) [22], Laboratory Department Services (Shaikh, 2017) [23], Emergency Department Services (Shaikh, 2017) [24].

\section{ZUBER'S CORONAVIRUS DISEASE (COVID- 19) STANDARDS FOR HOSPITALS :}

These standards are divided in to 17 Chapters and 160 Standards as follows:

1. Leadership - (12 Standards)

2. Facility Management and Safety- (10 Standards)

3. Staff, Mental Health and Well-Being- (18 Standards)

4. Prevention and Control of Infection- (36 Standards)

5. Emergency Room- (6 Standards)

6. Respiratory Triage Areas- (7 Standards)

7. Case Management- (13 Standards)

8. Housekeeping Services- (6 Standards)

9. Airborne Infection Isolation Room/ Isolation Ward- (3 Standards)

10. Logistics and Management of Supplies, Pharmaceuticals and Resources- (14 Standards)

11. Space Management- (5 Standards)

12. Incident Management System- (2 Standards)

13. Surge Capacity- (6 Standards)

14. Surveillance- Early Warning and Monitoring- (3 Standards)

15. Communication- (7 Standards)

16. Laboratory Services- (5 Standards)

17. Continuity of Essential Health, Support and Patient Care Services- (7 Standards)

\section{(1) LEADERSHIP:}

1.1 Hospital leaders should develop all the policies, procedures, guidelines, protocols, pathways to receive, handle, manage, transport and follow-up upon discharge of the COVID-19 Patients.

1.2 All these policies, procedures, guidelines, protocols, pathways, forms and Checklists should be developed based on (including but not limited to) the latest scientific references or evidence, best practices, national and international trends, Ministry of Health and World Health Organization recommendations.

1.3 The policies, procedures, guidelines, protocols, pathways should include special consideration for the management and treatment of COVID-19 Patients with co-morbidities.

1.4 Hospital leaders and Infection Control Practitioners should ensure that all approved Process FlowCharts and Protocols are accessible and visible to all Healthcare Workers for the Management and Treatment of COVID-19 Patients.

1.5 Hospital leaders should establish the Respiratory Clinics in the Emergency Department for the smooth and seamless management of all kinds of patients.

1.6 Hospital leaders should ensure that all the Healthcare Workers have received Education, Training and Competencies on COVID-19 Infection Control Practices.

1.7 Hospital leaders should list the required Personal Protective Equipment, supplies, medications, staff etc. and should ensure that are available and accessible to manage all routine and any influx. 
1.8 Hospital leaders and Infection Control Practitioners should ensure that all healthcare workers use only fit tested $\mathrm{N}-95$ respirators and all sizes and types of $\mathrm{N}-95$ respirators must be available at the health care area.

1.9 Hospital leaders and Infection Control Practitioners should ensure that all Aerosol Generating Procedures (AGP) on suspected or confirmed COVID-19 Patients are performed in a Negative Pressure Isolation Room.

1.10 Hospital leaders and Infection Control Practitioners should ensure that the hospital have required number of Airborne Infection Isolation Rooms as per the Ministry of Health or World Health Organization and or relevant International Accreditation Standards.

1.11 Infection Control Practitioners should ensure that the Isolation Signs and Symbols (Color Coded) are used at every stage of treatment, transfer of the patient including but not limited to patient rooms indicating the type of precautions required for staff and visitors.

1.12 Hospital leaders should conduct the Risk Assessment (by involving the Medical, Nursing, Paramedical, Ancillary, Administrative, Facility Management and Safety, Pharmaceutical, Infection Control, Quality etc.) for CORONA- 19 and develop the mitigation plan in order to mitigate all risks ahead of time on periodic basis.

\section{(2) FACILITY MANAGEMENT AND SAFETY:}

2.1 Hospital leaders and Infection Control Practitioners should ensure that all the HEPA filters are changed on a regular basis as per the recommendations of manufacturer and should be documented as per the hospital policy.

2.2 The distance that should be maintained between patients` beds are minimum of 2.4 meters in Critical Care Units.

2.3 The distance that should be maintained between dialysis chairs/beds are minimum of 1.2-1.5 meters.

2.4 There's at least one AIIR for each 8 bed in the Critical Care Unit (at least one even the number of beds less than 8 ).

2.5 At least, the unit has one AIIR (negative pressure room) or single room with portable HEPA filter / color coded sign according the policy.

2.6 Hospital leaders should ensure that the policy on visitors has been established and implemented.

2.7 Hospital leaders should maintain a log book for healthcare workers and visitors who had entered the isolation rooms in each shift on daily basis.

2.8 Hospital leaders should ensure that there is one negative pressure Airborne Infection Isolation Rooms (AIIRs) for every 25 to 30 in-patient admission beds is available.

2.9 Hospital leaders should ensure that all the Airborne Infection Isolation Rooms (AIIRs) has negative pressure of minimum -2.5 Pascal with a High-Efficiency Particulate Air (HEPA) filter to exhaust $100 \%$ of the air outside with air changes rate of at least $12 \mathrm{ACH}$ per hour. The assigned trained and competent staff should document these readings on daily basis as per the hospital policy. An audiovisual alarm to monitor the pressure difference, Air Changes per Hour, temperature and relative humidity should be fixed at the entrance of the room.

2.10 Hospital leaders should ensure that there must be a continuous monitoring for the ventilation parameters of the isolation room though a fixed monitor outside the room showing pressure difference, $\mathrm{ACH}$, temperature, relative humidity with audiovisual alarm when there is acute change in pressure difference.

\section{(3) STAFF, MENTAL HEALTH AND WELL-BEING:}

3.1 Hospital leaders should ensure that sufficient daily number of medical, nursing, para-medical, ancillary staff are available in each shift as per the volume, acuity and needs.

3.2 Hospital leaders should conduct a daily assessment of staffing status and needs.

3.3 A back-up staffing plan to determine minimum staffing needs to provide a safe work environment and safe patient care

3.4 Hospital leaders should assign dedicated staff for the care of COVID patients.

3.5 Hospital leaders should adhere to the hospital policy on end-of-life care patients for COVID patients.

3.6 Hospital leaders should develop the crisis capacity strategies for staff shortages and manage them accordingly. 
3.7 Hospital leaders should have a process to redeploy quality and non-clinical staff to support frontline teams for non-clinical activities and in the same way for medical and nursing staff to deploy for appropriate clinical and non-clinical activities.

3.8 Hospital leaders should have a policy and procedure to proactively manage fear and anxiety in daily work for all staff.

3.9 Hospital leaders should have a policy on sick leave that is non-punitive, flexible, and consistent with relevant regulatory authorities or public health policies.

3.10 Hospital leaders should support equitable financially to staff and their families should they need to be quarantined.

3.11 Hospital leaders should provide equitable transportation for medical and non-medical staff as needed to protect staff safety.

3.12 Hospital leaders should equitable access to staff "psychological PPE" to prevent, mitigate, and manage emotional and psychological distress.

3.13 Hospital leaders should update all staff contact list and monitor the staff absenteeism continuously in advance.

3.14 Hospital leaders should develop a policy and procedure to monitor and manage staff suspected or confirmed of having COVID-19 or who have had exposure to a confirmed, probable or suspected COVID-19 patient.

3.15 Hospital leaders should identify domestic support measures which could enhance staff flexibility for shift work and longer working hours and define off work time for recuperation.

3.16 Hospital leaders should provide multidisciplinary psycho-social support for the families of staff and patients.

3.17 Hospital leaders should address liability, insurance and temporary licensing issues with respect to staff who may be working outside their areas of expertise.

3.18 Hospital leaders should ensure that there are approved policies are in place to manage volunteer workers.

\section{(4) PREVENTION AND CONTROL OF INFECTION:}

4. 1 The Prevention and Control of Infection Department should have personnel qualified in Infection Control by education, license, training and experience as per the requirements of national laws, regulations, norms and requirements.

4. 2 The hospital should have minimum one full time Infection Control Practitioner for each 100 beds, 30 Critical Care beds and 120 dialysis patients in Dialysis Unit.

4. 3 All the Infection Control Practitioners should have the current scientific (national and international level) knowledge on COVID-19 diagnosis, management, precautions, trends and outcome.

4. 4 Hospital should have adequate resources (staff, supplies, equipment, medications, technology, space, stationary, oxygen and supplies required for the same etc.)

4. 5 Hospital leaders should formulate outbreak management team to manage the disaster and should conduct the drills for testing and system improvement on every six monthly basis to ensure it effectiveness.

4. 6 Hospital leaders should have Terms of References of Infection Prevention and Control Committee which should be chaired by the Infectious Disease Consultant on minimum monthly basis.

4. 7 Hospital leaders should select the Quality Measures to be monitored for COVID-19 and monitor on periodic or monthly basis and should be discussed in the hospital Infection Prevention and Control Committee.

4. 8 Hospital leaders should conduct the daily Infection Prevention and Control rounds with the identified members and document the observations for action plan and to monitor the progress.

4. 9 Hospital leaders should provide education to all the health care workers (HCW), patients and visitors on prevention of healthcare-associated infections, respiratory care and hand hygiene.

4. 10 Hospital leaders should provide verbal instructions, informational posters, cards, etc. in national and international languages to all patients, families, visitors etc.

4. 11 Hospital leaders should install hand hygiene stations and waste bins at strategic locations across the hospital facility. 
4. 12 Hospital leaders should ensure that all healthcare workers are adhering to the standard, contact, droplets, and airborne precautions as per the case of the patients. These precautions should be continued until the patient is asymptomatic and negative swab culture.

4. 13 Hospital leaders should place the patients in adequately ventilated single rooms $(60 \mathrm{~L} / \mathrm{s}$ per patient). When single rooms are not available, patients suspected of having COVID-19 should be grouped together and should not mix the suspected and confirmed cases.

4. 14 Hospital leaders should ensure that a distance of one-meter between the two beds regardless of whether patients are suspected of having COVID-19 is maintained.

4. 15 Hospital leaders should ensure that used equipment are either for single-use and disposable or if equipment needs to be shared among patients, should be cleaned and disinfect between use for each patient by using ethyl alcohol $70 \%$, etc.

4. 16 Hospital leaders should ensure that the housekeeping staff are routinely cleaning and disinfecting surfaces with which the patient is in contact and implanting methods of routine cleaning and disinfection of ambulances as per the national or international guidelines.

4. 17 Hospital leaders should ensure that the healthcare workers are applying airborne precautions for aerosol-generating procedures.

4. 18 Hospital leaders should ensure that where possible, a team of healthcare workers is designated to care exclusively for suspected or confirmed cases in order to reduce the risk of transmission.

4. 19 Hospital leaders should ensure that all staff received training on standard, contact, droplets, and airborne precautions (including correct use of PPE, donning and doffing, masks tested for fitting, hand hygiene, respiratory hygiene, etc.). Evidences of documents or records should be readily available and accessible.

4. 20 Hospital leaders should ensure that sufficient Personal Protective Equipment (PPE) including but not limited to N95 masks of all different sizes, surgical masks, FFP2 respirators, face shield, eye protection goggles, gloves and gowns are easily available and accessible to staff. In the event of shortage PPE's, the hospital leaders should prioritize for the staff who are involved in the patient care.

4. 21 Hospital leaders should avoid moving and transporting patients out of their room or area unless medically necessary.

4. 22 Hospital should have a designated portable X-ray equipment and/or other designated diagnostic equipment. If transport is required, predetermined transport routes should be used to minimize exposure for staff, other patients, visitors, and the patient should use a medical mask if tolerable or reinforce respiratory hygiene.

4. 23 Hospital leaders should ensure that healthcare workers who are transporting patients perform hand hygiene and wear appropriate PPE and notify the area receiving the patient of any necessary precautions as early as possible before the Patient's arrival.

4. 24 Hospital leaders should limit visitors to those essential for patient and support.

4. 25 Hospital leaders should manage laboratory specimens, laundry, foodservice utensils, and medical waste following safe routine procedures according to Infection Prevention and Control Guidelines.

4. 26 Hemodialysis unit should have clear pathways and flowcharts to handle the patients with respiratory illness suspected cases.

4. 27 Hospital should have a system for screening, testing, and reporting COVID-19 symptoms among staff, patients, and visitors which should include but not limited to:

a) Standardized process to screen patients and staff prior to arriving at the facility for symptoms compatible with COVID-19 and exposure to individuals with COVID-19 up to 14 days prior to coming to the facility.

b) Standardized process to screen patients, visitors, and staff upon arrival at the facility for symptoms compatible with COVID-19 and exposure to individuals with COVID-19 up to 14 days prior to coming to the facility.

c) Confirmation that they are asymptomatic

d) Temperature check

e) SARS-CoV-2 test (RT-PCR, antigen, or other test), if indicated

f) Standardized process for all staff to self-screen for COVID-19 symptoms and exposures prior to every shift (e.g., attestation, temperature screenings) 
g) Standardized protocol to refer patients who screen positive for symptoms

h) Immediate notification to facility leadership and local or state health department

i) System to track admissions and discharges.

4. 28 Hospital should have a system for screening, testing, and reporting COVID-19 symptoms among community members across all local neighborhoods, equitably.

4. 29 Hospital should have clear entry pathways for access into respiratory support.

4. 30 Hospital should have protocol for isolation of exposed or infected health care personnel and patients.

4. 31 Hospital should have a system for verifying that staff are competent in the actions/skills for which they are being trained.

4. 32 Hospital should have a documented policy for visitor access and movement which should include but not limited to:

a) Guidelines for social distancing.

b) Limitations and restrictions into the rooms of patients with suspected or confirmed COVID19.

c) Criteria when entering the room of a patient.

d) Remote communication between patient and visitor.

4. 33 Hospital should have a policy and procedure for management of postmortem care and disposition of deceased COVID patients which should include but not limited to:
a) PPE requirements
b) Storage procedures
c) Cleaning and waste disposal
d) Plans for expanding morgue capacity

4. 34 Hospital should have a policy and procedure for food supply and management which should include but not limited to:

a) Personal hygiene requirements

b) Supplies needed

c) Instructions for cleaning, including frequency

d) Procedures for accepting deliveries

e) Procedures for food storage and production

f) Details on how long the hospital can operate and feed essential workers (where necessary)

4. 35 Hospital should continuing other, necessary non-COVID-19 Care.

Tiered surge criteria, including metrics (e.g., to determine when to cancel elective admissions and surgeries).

a) A plan for prioritizing and allocation of health care services (crisis standards of care).

b) A plan for reducing or eliminating face-to-face encounters (e.g., assessment of what is suitable for temporary/remote work).

c) A plan for maximizing the use of telemedicine for patients, family members, and virtual meetings for staff.

4. 36 Hospital leaders should ensure that the assessment of patients with Acute Respiratory Infections is done as per updated COVID-19 guidelines.

\section{(5) EMERGENCY ROOM:}

5.1 Hospital leaders and Infection Control Practitioners should ensure that the written reminders for updated definitions of suspected cases of COVID-19 is available and accessible in the Emergency Department and all Clinical Staff are quite familiar with these definitions.

5.2 Hospital leaders and Infection Control Practitioners should ensure that the nasopharyngeal swabbing and sputum sampling of suspected patients is performed by trained Clinical Staff 24/7.

5.3 Hospital leaders and Infection Control Practitioners should ensure that a logbook is maintained for all healthcare workers and visitors who had entered the Airborne Infection Isolation Rooms.

5.4 Hospital leaders and Infection Control Practitioners should ensure that a standard checklist is implemented to monitor and implement the parameters of the Airborne Infection Isolation Rooms in each shift on all days.

5.5 Hospital leaders and Infection Control Practitioners should ensure that the distance between the two beds is 1.2 meter in Emergency Room 
5.6 Hospital leaders and Infection Control Practitioners should ensure that preferably a dedicated portable chest x-ray is available for the usage in Respiratory Clinics in Emergency Department or Airborne Infection Isolation Rooms as and when needed.

(6) RESPIRATORY TRIAGE AREAS:

6.1 All entrances of the hospital should have a designated area for respiratory triage with required equipment including but not limited to surgical facemask, hand hygiene sanitizer, posters, face shield, etc.

6.2 All the visual triage areas of the hospital should be covered by trained and competent nursing staff who is able to communicate with patients in both local/ national and English languages.

6.3 Latest, standard and approved respiratory triage forms should be used as per the Ministry of Health and or World Health Organization.

6.4 Based on the scores of respiratory triage all the patients should be advised for wearing personal protective equipment and hand hygiene and or directed safely to respiratory waiting area.

6.5 Hospital should have designated respiratory waiting or triage areas with:

a) Fixed chairs (preferably with plastic covers in order to clean it frequently)

b) arranged by a safe distance of at least 1.2 meter

c) Posters in local/ national and English language on use of respiratory hygiene PPE's.

d) Trash bin with lid as per the Infection Control Guidelines.

e) Hand sanitizers and tissue papers.

f) Chairs to accommodate obese and physically challenged patients.

g) Culture, values and beliefs should be respected.

6.6 Qualified physicians by education, licenses, experience and training should be available at Respiratory Triage Clinics, Airborne Infection Isolation Rooms etc. to assess and treat patients based on the case definitions of CORONA-2019.

6.7 Hospital leaders should transfer all suspected patients to Airborne Infection Isolation Rooms for necessary chest $\mathrm{x}$-ray and swabbing.

\section{(7) CASE MANAGEMENT:}

7.1 Hospital leaders should designate an exclusive well ventilated, low-transit, and secure waiting and examination area for individuals presenting with respiratory symptoms and/or fever.

7.2 Medical and Nursing staff assigned for Triage area should ensure that the patients with symptoms of respiratory distress and severe underlying conditions are prioritized for medical Evaluation.

7.3 Hospital leaders should identify a place outside or within the premises of the hospital for establishing a triage area for patients presenting to the hospital.

7.4 All triage areas should be under the supervision of a responsible and accountable triage supervisor for overseeing all triage operations.

7.5 Hospital leaders should follow the triage protocol as per the national or WHO Standards for all cases of acute respiratory infection.

7.6 Hospital leaders should ensure that all suspected cases of COVID-9 are maintaining sufficient distance in space that is assigned to them.

7.7 Hospital leaders should ensure that the application of standard, and droplet precautions are maintained at all times.

7.8 Hospital leaders should implement the hospital strategy for the admission, internal transfer, referral, and discharge of Severe Acute Respiratory Illness (SARI) patients, in line with relevant criteria and operational protocols in coordination with local health authorities.

7.9 Hospital leaders should guide the patients for home care for mild cases of COVID-19 acute respiratory infection in individuals with no comorbidities, recognized as posing a risk for severe or fatal disease associated with COVID-19.

7.10 Hospital leaders should consider admission for cases of COVID-19 acute respiratory infection with comorbidities recognized as posing a risk for a severe or fatal course of COVID-19.

7.11 Hospital leaders should ensure that all the available beds are staffed for the admission of severe COVID-19 acute respiratory infection cases. 
7.12 Hospital leaders should continuously monitor the vital signs (e.g., temperature, blood pressure, pulse, respiratory rate, level of consciousness, clinical signs of dehydration or shock), and oxygen saturation (pulse-oximetry or blood gas analyses) and document in the medical records as per the hospital policy.

7.13 Hospital leaders should ensure the availability of oxygen and means of respiratory support, sedation medications and supplies for intubated patients. Oxygen masks and nasal canulae should be singleuse.

\section{HOUSEKEEPING SERVICES:}

8.1 Housekeeping Manager or Supervisor should develop the housekeeping services policies, procedures, guidelines, protocols, pathways forms, Checklists etc. based on (including but not limited to) the latest scientific references or evidence, best practices, national and international trends, Ministry of Health and World Health Organization recommendations.

8.2 Hospital leaders and Infection Control Practitioners should ensure that the chemicals used for the housekeeping services are approved by the national regulatory authorities.

8.3 Housekeeping Manager or Supervisor should ensure that all the resources (chemicals, trolleys, spill kits, PPE's, soap, water, hand sanitizers, hand washing sinks, surface disinfectants, alcoholic hand rub dispenser etc.) required for housekeeping are available to manager the operations in normal and influx conditions.

8.4 Hospital leaders and Infection Control Practitioners should ensure that there is a cleaning and or disinfection schedule and activities logbook is maintained.

8.5 The cleaning and or disinfection schedule and activities logbook should include the parameters such as housekeeper's name, Identification Number, used chemicals, cleaning method and frequency and checklist of environmental surfaces cleaning including lockers, cabinets, furniture and fixtures are regularly cleaned, dry and dust free.

8.6 Housekeeping Supervisor should ensure that the terminal cleaning process is done properly when indicated by using detailed checklist and supervised by the in-charge nurse.

\section{(9) AIRBORNE INFECTION ISOLATION ROOM/ ISOLATION WARD:}

9.1 Biological spill kits should be available and accessible in all the identified areas and all responsible staff should demonstrate appropriate technique for management of blood and/or body fluids.

9.2 Hospital leaders should ensure that the appropriate education material in national and English Language is available and accessible for all staff, patients, visitors and trainees for all infection control practices and procedures.

9.3 Hospital leaders should ensure that a portable chest $\mathrm{x}$-ray is available for usage in isolation room when needed.

\section{(10) LOGISTICS AND MANAGEMENT OF SUPPLIES, PHARMACEUTICALS AND} RESOURCES:

10.1 Hospital leaders should maintain the continuity of hospital services.

10.2 Hospital leaders should develop and maintain inventory of (including but not limited to) all equipment, supplies, staff, and pharmaceuticals etc.

10.3 Hospital leaders should estimate the consumption of essential equipment, supplies, and pharmaceuticals per day or per week based on most likely outbreak scenario.

10.4 Hospital leaders should always check with national or local authorities to ensure the continuous provision of essential medications and supplies.

10.5 Hospital leaders should assess the quality of contingency items prior to the purchase and should request quality certification.

10.6 Hospital leaders should ensure that there is a stable source of supplies and at least eight weeks of essential patient care equipment (e.g., pumps, ventilators) and PPE (e.g., face and surgical masks, face shields, respirators, gowns, gloves, eye protection, hand sanitizer, disinfectant, body bags etc.

10.7 Hospital leaders should have a process to regularly calculate and communicate the hospital's daily PPE usage rate. 
10.8 Hospital leaders should have a contingency plan for decontamination, substitution, or reuse/extended use in case of supply shortages.

10.9 Hospital leaders should communicate with all suppliers and authorities to ensure the continuous provision of essential medications and supplies.

10.10 Hospital leaders should also allocate the physical space within the hospital premises as the required standards and regulatory requirements or guidelines for the storage of supplementary supplies.

10.11 Hospital leaders should follow the standard materials management guidelines for the management of all stocks.

10.12 Hospital leaders should develop and implement the policy on supplying medications and supplies for the cases treated at home or other alternative treatment sites.

10.13 Hospital leaders should ensure that all maintenance and repair of the equipment should be done on urgent basis.

10.14 Hospital leaders should develop a policy for managing donations of medical supplies, food for staff, etc.

\section{(11) SPACE MANAGEMENT:}

11.1 Hospital leaders should identify the space in Emergency Department, Critical Care Units and other patient care areas for separation of known or suspected COVID patients.

11.2 Hospital leaders should have a process in place to communicate or contact the patients and families by phone in the absence of beds or space in the hospital and a process to allow patients to wait in a personal vehicle or outside the facility.

11.3 Hospital leaders should identify spaces with surge capacity capabilities for their hospital.

11.4 Hospital leaders should have a plan for allocating negative pressure rooms.

11.5 Hospital leaders should have a plan for High Efficiency Particulate Air (HEPA) filters to convert regular rooms into ones with negative air pressure.

\section{(12) INCIDENT MANAGEMENT SYSTEM:}

12.1 Hospital leaders should have a well-functioning Incident Management System for the effective management of emergency operations. The Incident Management System should be developed based on the relevant national or regulatory standards or international references.

12.2 Hospital Incident Management System should include but not limited to the below members:

a) Hospital Chief Executive Officer

b) Hospital Director

c) Nursing Director

d) Quality Director

e) Information and Technology Director

f) All Clinical Heads of the Departments

g) Infection Prevention and Control Director

h) Respiratory Therapist

i) Human Resources Manager

j) Security Supervisor

k) Pharmaceuticals Manager

1) Clinical Engineering

m) Laboratory Services Director

n) Radiology Services Director

o) Dietary Services Manager

p) Laundry Services Manager

q) Cleaning and Waste Management Manager

r) Supply Chain Management Director

s) Social Worker

t) Patient Experience Manager

\section{(13) SURGE CAPACITY:}


13.1 Hospital leaders should calculate maximal case admission capacity by the total number of beds, availability of manpower, space, critical care beds, isolation wards or beds, cohort, the number of mechanical ventilators and the availability of other resources and supplies.

13.2 Hospital leaders should identify ways of expanding hospital in-patient capacity including but not limited to physical space, staff, supplies, medications and processes.

13.3 Hospital leaders should identify the gaps in the provision of health care and address these gaps in coordination with the authorities and neighboring hospitals.

13.4 Hospital leaders should outsource care of non-critical patients to other alternative healthcare facility or treatment sites in order to improve the hospital capacity.

13.5 Hospital leaders should identify additional sites for conversion to patient care units in coordination with the local authorities.

13.6 Hospital leaders should temporarily cancel nonessential services in order to improve efficiency.

\section{(14) SURVEILLANCE- EARLY WARNING AND MONITORING:}

14.1 Hospital leaders should appoint a qualified epidemiologist by education, license, experience and training.

14.2 Hospital leaders should define the information to be collected on periodic basis.

14.3 Hospital leaders should report all incidents related to COVID-19 by health-care workers to the hospital and to the ministry of health (if requested).

\section{(15) COMMUNICATION:}

15.1 Hospital leaders should ensure that the technology and resources are available to support the mechanisms of communication in order to streamline the sharing of information between the leaders, colleagues, government or regulatory authorities along with the updated contact list with roles.

15.2 Hospital leaders should have cards of roles and responsibilities for all the responsible staff.

15.3 All information materials in paper or digital form should be easily available and accessible to all staff.

15.4 Hospital leaders should collect all the information as per the requirements of the local regulatory authorities, analyze and submit to them and also take decisions based on the information or data.

15.5 Hospital leaders should publish the data or information in media for the awareness of patients, visitors, staff and the general public.

15.6 Hospital leaders should select a spokesperson to communicate with the public, the media, and local health authorities on periodic basis.

15.7 Hospital leaders should have the list of healthcare facilities for referrals and transfers as per the need and clinical condition of the patient.

(16) LABORATORY SERVICES:

16.1 Hospital and Laboratory leaders should ensure that the continuous availability of basic laboratory testing (e.g., complete blood count, biochemistry profile, electrolytes, blood gas analysis, blood culture, and sputum examination).

16.2 Hospital and Laboratory leaders should have the back-up of all essential supplies, resources and personnel of laboratory in order to maintain the continuity.

16.3 Hospital and Laboratory leaders should have the technology and resources to release the reports as per the standard required time and hospital policy.

16.4 Hospital and Laboratory leaders should follow all guidelines as per the ministry of health or relevant regulatory authorities.

16.5 Hospital and Laboratory leaders should train all staff on all processes of the laboratory.

\section{(17) CONTINUITY OF ESSENTIAL HEALTH, SUPPORT AND PATIENT CARE SERVICES:}

17.1 Hospital leaders should identify the food supplies in order to ensure the food is available in all critical conditions for staff, patients, families etc.

17.2 Ensure the availability of appropriate back-up arrangements for essential life-lines including but not limited to water, power, and oxygen based on the risk assessment. 
17.3 Hospital leaders should control all the accesses of the hospital to maintain the essential pharmaceutical stocks, patient flow, traffic, and parking etc.

17.4 Hospital leaders should allocate a physical space for the temporary morgue use and should also ensure that the adequate body bags and shroud packs are available.

17.5 Hospital leaders should identity the list of services based on the priority.

17.6 Hospital leaders should provide all identified services in order to maintain the hospital operations at all times and under any circumstances.

17.7 Hospital leaders should identify all resources as per the need in order to maintain the continuity of care.

\section{FURTHER RESEARCH :}

Further research is needed to understand the impact of Zuber's Coronavirus Disease (COVID- 19) Standards for Hospitals.

\section{PRACTICE IMPLICATIONS :}

The healthcare providers should implement Zuber's Coronavirus Disease (COVID- 19) Standards for Hospitals in order to fight the COVID-19.

\section{CONCLUSION :}

The study concluded with "Zuber's Coronavirus Disease (COVID- 19) Standards for Hospitals."

\section{LIMITATIONS OF THE STUDY :}

Coronavirus Disease (COVID- 19) is the only limitation of this study.

\section{DIRECTIONS FOR FUTURE RESEARCH :}

In the future, research should be conducted to study the overall impact of Zuber's Coronavirus Disease (COVID- 19) Standards for Hospitals in all types of hospitals.

\section{SOURCES OF FUNDING FOR THE STUDY :}

This study was self-funded by the author.

\section{DISCLAIMER :}

This publication contains information obtained from authentic and highly regarded sources. Reasonable effort has been made to publish reliable data and information, but the author and publisher cannot assume responsibility for the validity of all materials or for the consequences of the use.

All rights reserved. No part of this publication may be reproduced, stored in a retrieval system or transmitted, in any form, or by any means, electronic, mechanical, photocopying, recording or otherwise, without permission, in writing, from the publisher or the author.

\section{REFERENCES :}

[1] World Health Organisation. Timeline - COVID-19. https://www.who.int/coronavirus/timeline

[2] Huang C, Wang Y, Li X, et al. (2020). Clinical features of patients infected with 2019 novel coronavirus in Wuhan, China. Lancet, 395(10223), 497-506.

[3] McMahon DE, Peters GA, Ivers LC, Freeman EE (2020). Global resource shortages during COVID-19: Bad news for low-income countries. PLoS Negl Trop Dis, 14(7), e0008412.

[4] Livingston E, Desai A, Berkwits M. (2020). Sourcing Personal Protective Equipment During the COVID-19 Pandemic. JAMA.

[5] Ranney M. L, Griffeth V, Jha A. K. (2020). Critical Supply Shortages-The Need for Ventilators and Personal Protective Equipment during the Covid-19 Pandemic. New England Journal of Medicine, 382(18), e41.

[6] Stefan, T., \& Nazarov, A. D. (2020, November). Challenges and competencies of leadership in Covid- 
19 Pandemic. In Research technologies of pandemic coronavirus impact (RTCOV 2020) (pp. 518-524). Atlantis Press.

[7] Rau J. Coronavirus Stress Test: Many 5-Star Nursing Homes Have Infection-Control Lapses. Kaiser Health News. (March 4, 2020)

[8] Condon, B \& Herschaft, R. (April 13, 2020).

[9] Ingold, J. (2020). Many Colorado nursing homes were already struggling to control infections. Then came coronavirus. The Colorado Sun. Retrieved from https://coloradosun.com/2020/04/08/coloradonursing-home-infection-coronavirus/

[10] Harrington C, et al. (2012). Nurse staffing and deficiencies in the largest for-profit nursing home chains and chains owned by private equity companies. Health services research, 47(1), 106-128.

[11] Williams C, Zheng Q, \& White A. (2019). Payroll-Based Staffing Measures for Nursing Homes. Innovation in Aging., 3(Suppl 1), S62.

[12] Harrington C, et al. (2016). Article commentary: The need for higher minimum staffing standards in US nursing homes. Health services insights, 9(1), S38994.

[13] Pineles L, et al. (2019). Frequency of nursing home resident contact with staff, other residents, and the environment outside resident rooms. Infection Control \& Hospital Epidemiology, 40(7), 815-6.

[14] Rau J. Coronavirus Stress Test: Many 5-Star Nursing Homes Have Infection-Control Lapses. Kaiser Health News.

[15] Kavanaugh, K. (2020). As COVID-19 Knocks on Nursing Homes' Doors, Full-Time Infection Preventionists Needed More Than Ever. Infection Control Today. Retrieved from https://www.infectioncontroltoday.com/view/when-covid-19-knocks-nursing-homes-doors-systemicproblems-welcome-it

[16] CDC: Life Care staff worked in multiple facilities and while symptomatic for COVID-19. Seattle Weekly. March 19, 2020. Retrieved from https://www.seattleweekly.com/news/cdc-life-care-staffworked-in-multiple-facilities-and-while-symptomatic-for-covid-19/

[17] Afsoon Asadzadeh, Saba Pakkhoo, Mahsa Mirzaei Saeidabad, Hero Khezri, Reza Ferdousi, (2020). Information technology in emergency management of COVID-19 outbreak. Informatics in Medicine Unlocked, 21(1), 100475,

[18] Georgios Peros, Ferda Gronki, Nadine Molitor, Michael Streit, Kiyoshi Sugimoto, Urs Karrer, Fabian Lunger, Michel Adamina, Stefan Breitenstein \& Tenzin Lamdark (2020). Organizing a COVID-19 triage unit: a Swiss perspective. Emerging Microbes \& Infections, 9(1), 1506-1513.

[19] Bikkina, S., Kittu Manda, V., \& Adinarayana Rao, U. V. (2021). Medical Oxygen Supply During COVID-19: A Study with Specific Reference to State of Andhra Pradesh, India. Materials today. Proceedings, 10.1016/j.matpr.2021.01.196. Advance online publication.

[20] Shaikh, Z. M. (2016). The impact of hospital accreditation on the ambulance services satisfaction. International Journal of Emerging Research in Management \& Technology (IJERMT), 5(12), 76-84.

[21] Shaikh, Z. (2017). The Impact of Hospital Accreditation on the Patient's Satisfaction of physical therapy Department Services. International Journal of Business, Management and Allied Sciences (IJBMAS), 4(4.2017), 143-154.

[22] Shaikh, Z. M. (2017). The Impact of Hospital Accreditation on the Patients Satisfaction of Dietary Services. International Journal of Business, Management and Allied Sciences (IJBMAS), 4(4.2017), 112.

[23] Shaikh, Z. M. (2017). The Impact of Hospital Accreditation on the Patients Satisfaction of Laboratory Department Services. International Journal of Business, Management and Allied Sciences (IJBMAS), 4(2), 4277-4289. 
[24] Shaikh, Z. M. (2017). The Impact of Hospital Accreditation on the Patients Satisfaction of Emergency Department Services. International Journal of Business, Management and Allied Sciences (IJBMAS), 4(3), 4330-4339. 